\title{
Wie wird die RSV-Prophylaxe in Deutschland angenommen?
}

\section{How is the RS-Virus Prophylaxis in Germany Adopted?}

J. Forster, R. Urbanek

Bibliography

Dol http://dx.doi.org/ 10.1055/s-0031-1283208

Klin Padiatr 2011; 223: 257-258

(c) Georg Thieme Verlag KG

Stuttgart · New York

ISSN 0300-8630

Korrespondenzadresse

Prof. Dr. Johannes Forster, MME (Bern)

Kinderabteilung St. Hedwig

St. Josefskrankenhaus

Sautierstraße 1

79104 Freiburg i. Br.

paediatrie@rkk-sjk.de

Prof. Dr. Radvan Urbanek

Zentrum für Kinder- und

Jugendmedizin

Mathildenstraße 1

79106 Freiburg i. Br.

rurb@gmx.at
Obwohl eine Infektion mit Respiratory-SyncitialVirus (RSV) als Infekt der oberen Atemwege beginnt, verbreiten sich die Viren bei Säuglingen und jungen Kindern schnell in die peripheren Abschnitte der unteren Atemwege und verursachen das schwere Krankheitsbild einer Bronchiolitis und /oder einer Pneumonie. RSV ruft die meisten Infektionen der unteren Atemwege in den ersten 2 Lebensjahren hervor, in den USA werden mit dieser Diagnose von 1000 Säuglingen 3-9 im 1. Lebensjahr hospitalisiert.

Unter unseren klimatischen Bedingungen finden epidemische RSV-Infektionen in den Monaten September/Oktober bis April/Mai statt. Die Infektiosität ist hoch, man schätzt, dass während der Saison etwa $20 \%$ der Kinder bereits nosokomial infiziert werden, weshalb man schon vor der Verlegung der Risikokinder von der Intensivpflegestation auf eine normale Station oder vor Entlassung nach Hause auf den Aufbau eines immunologischen Schutzes drängt. Wenn auch fast alle Kinder in den ersten 2 Lebensjahren die RSVInfektion durchgemacht haben, kommen wiederholte RSV-Infektionen in späterem Alter vor. Bei älteren Kindern und Erwachsenen stehen leichtere Symptome der oberen Atemwege im Vordergrund, im Greisenalter werden die Verläufe erneut schwerer.

Sowohl Morbidität als auch die Mortalität ist in den Risikogruppen „sehr unreife Frühgeborene mit bronchopulmonaler Dysplasie, junge Kinder mit angeborenen Herzfehlern, Kinder mit neuromuskulären Erkrankungen oder mit chronischen Immundefekten und Kinder mit pulmonalen Erkrankungen wie zystische Fibrose und Ziliendyskinesie“ hoch, sodass vordringlich für diese Risikogruppen effektive Präventivmaßnahmen in den ersten Lebensjahren angestrebt werden.

Bisherige Studien mit intravenösem RSV-Immunglobulin haben mit Blick auf die Hospitalisierung keine Überlegenheit dieses Vorgehens im Vergleich zur alleinigen supportiven Therapie belegen können. Demgegenüber konnte 1998 in der nordamerikanischen Studie „Impact“ [1] mit monatlichen intramuskulären Injektionen von Palivizumab bei sehr unreifen Frühgeborenen mit und ohne bronchopulmonaler Dysplasie und in einer nachfolgenden Untersuchung [3] auch bei Kindern mit kongenitalen hämodynamisch wirksamen Herzfehlern die Rehospitalisation auf etwa die Hälfte [8] reduziert werden. Dieser humanisierte monoklonale Antikörper bindet das Fusionsprotein des RS-Virus und behindert damit das intrazelluläre Eindringen.
Wenn sich auch die theoretische Frage stellt, ist der Krankheitsverlauf infolge der passiven Immunisierung milder oder handelt es sich um eine spezifische Prophylaxe, haben die DGPI, DGPK, DPP und die GNPI eine gemeinsame Empfehlung verabschiedet [4], welche Kinder prophylaktisch behandelt werden sollen. Ziegler und Straßburg [10] berichten, dass von 318 Kinder mit Geburtsgewicht unter $1500 \mathrm{~g}$ in Deutschland nur 38,1\% bis zum Alter von 2 Jahren Palivizumab erhalten hatten. Die berichteten Daten lassen nicht erkennen, auf welcher individuellen Entscheidungsgundlage die Prophylaxe gegeben oder für nicht notwendig erachtet worden war.

Von Simon et al. [9] werden nun Daten einer großen deutschen multizentrischen Beobachtungsstudie über die Akzeptanz und erzielte Wirkung der Immunprophylaxe mit Palivizumab vorgelegt. Der Datenrücklauf war geringer als bei den ESPED-Erhebungen, bei denen wegen der Seltenheit der Erkrankungen mit einem höheren Interesse aller Teilnehmer gerechnet werden kann [5]. Die Autoren berichten über 49608 Antikörperinjektionen bei 10686 Kindern. Etwas mehr als ein Drittel (35\%) erhielt in der Infektionssaison insgesamt mehr als 5 monatliche Antikörpergaben. Die erste Injektion erfolgte noch in der Klinik, die Fortsetzung dann ambulant bei einem weiter betreuenden Arzt, nachdem die Eltern vor der Entlassung im Krankenhaus vom Neonatologen aufgeklärt wurden.

Schwere Nebenreaktionen waren selten, ein möglicher Zusammenhang mit der Antikörpergabe bestand nur in 6 von 10686 untersuchbaren Patienten (0,06\%), davon starb ein Kind an SIDS, ein weiteres Kind an einem nicht diagnostizierten fieberhaften Infekt und ein Kind mit einem Herzfehler an einem Herzstillstand bei ventrikulärer Arrhythmie, eine Anaphylaxie wurde in keinem Fall diagnostiziert.

Die Anzahl der untersuchbaren Kinder fiel im Verlaufe der mehrjährigen Studie ab, zu Beginn (im Jahre 2002/2003) betrug sie 26\%, bei ihrem Ende (2006/2007) nur noch $13 \%$; es ist anzunehmen, dass die umfangreiche Administration und Budgetsicherung hierfür verantwortlich war. Bewertete man nämlich die Adhärenz der Eltern zu dem Behandlungsplan der Immunisierung, so war sie als sehr gut bei $73 \%$, gut bei $18 \%$, mäßig bei $6 \%$ und als schlecht bei nur $3 \%$ eingestuft worden.

Insgesamt 324 Säuglinge von 9833 (3,3\%) wurden stationär in den 5 Perioden mit RSV-Infektion (Kalendermonate September bis Mai 20022007) aus unterschiedlichsten Gründen stationär aufgenommen. Die allerdings nicht bei allen Pa- 
tienten vollzogenen Virusnachweise bestätigten eine RSV-Infektion bei 156 Patienten, d.h. eine Rate von 2,5\% spezifischer RSVErkrankungen bei Kindern, die ab der postpartalen Entlassung Palivizumab erhielten. Jedenfalls wurden 111 (33\%) der wiederaufgenommenen Kinder intensivmedizinisch behandelt, 57\% benötigten eine erhöhte Sauerstoffgabe und 44 (13\%) wurden sogar beatmet.

Als zusätzliche Risiken zur Frühgeburtlichkeit waren von Döhring et al. [2] die bronchopulmonale Dysplasie und ein Geschwisterkind im Kindergarten- oder Schulalter ausgemacht worden. Auch die Daten von Simon et al. veranschaulichen die klinisch relevante Morbidität der Risikopopulation und rechtfertigen die aufwändigen Maßnahmen, die hier eine RSV-Infektion verhindern oder ihren Verlauf abmildern sollen.

In Übereinstimmung mit Gross et al. wurde ferner bestätigt, dass die meisten „Therapieversager“ bereits in dem Zeitabschnitt der beiden ersten Palivizumab-Injektionen vorkommen. Offensichtlich wird der volle passive Schutz erst später erreicht, was eindeutig für anfänglich kürzere Injektionsintervalle bzw. höhere Antikörpergaben zu Beginn spräche - so ergibt sich auch für dies Medikament, das zwar generell eine Zulassung für Frühgeborene besitzt, eine Off-label-Indikation [7].

Eine weitere Bemühung, die Immunprophylaxe zu verbessern, war auch die Entwicklung eines neuen Schutzantikörpers (Motavizumab). Weil stärkere, vor allem lokale, aber auch systemische Reaktionen beobachtet wurden, hat man die vorerst nur in USA beabsichtigte Produktanmeldung im Dezember 2010 zurückgezogen.

Natürlich wird es auch weiterhin nicht gelingen, alle Kinder vor RSV-Infektion zu schützen, aber schon die zunehmende Erkenntnis, dass prophylaktisch behandelte Säuglinge mit Unreife <35 Schwangerschaftswochen und zusätzlichen Risikofaktoren wie bronchopulmonale Dysplasie, kongenitale Herzfehler, angeborene Immunschwäche und neuromuskuläre Erkrankungen seltener aufgenommen und auch seltener intensivmedizinisch behandelt werden müssen, macht die monatlichen Antikörpergaben in dem Zeitraum einer RSV-Epidemie sinnig.

\section{Literatur}

1 The Impact-RSV Study Group. Palivizumab, a humanized respiratory syncytial virus monoclonal antibody, reduces hospitalization from respiratory syncytial virus infection in high-risk infants. Pediatrics 1998; 102: 531-537

2 Doering G, Gusenleitner W, Belohradsky BM et al. The risk of Respiratory Syncytial Virus related hospitalizations in preterm infants of 29 to 35 weeks gestational age. Pediatr Infect Dis J 2006; 25: 1188-90

3 Feltes TF, Cabalka AK, Meissner HC et al. Cardiac Synagis Study Group. Palivizumab prophylaxis reduces hospitalization due to respiratory syncytial virus in young children with hemodynamically significant congenital heart disease. J Pediatr 2003; 143: 532-540

4 Forster $J$, Herting $E$, Liese $J$ et al. Stellungnahme zur Prophylaxe von schweren RSV-Erkrankungen bei Risikokindern mit Palivizumab Deutsche Gesellschaft für Pädiatrische Infektiologie, Deutsche Gesellschaft für Pädiatrische Kardiologie, Gesellschaft für Pädiatrische Pneumologie, Gesellschaft für Neonatologie und Pädiatrische Intensivmedizin; AWMF-Leitlinien-Register Nr. 048/012http://www.awmf. org/uploads/tx_szleitlinien/048-012_S2k_Prophylaxe_von_schweren_ RSV-Erkrankungen_bei_Risikokindern_mit_Palivizumab_07-2010_ 07-2011.pdf; 22.6.2011)

5 Göbel U, Heinrich B, Krauth KA. Et al. Evaluation der Prozess- und Ergebnisqualität der Erhebungseinheit für seltene pädiatrische $\mathrm{Er}$ krankungen in Deutschland (ESPED). Klin Padiatr 2010; 222: 92-97

6 Gross $M$, Brune $T$, Jorch $G$ et al. Significance of respiratory syncytial virus (RSV) infection in the $1^{\text {st }}$ year of life. Infection 2000; 28: 34-37

7 Roth B. Off-label-use und Anwendung von unlizenzierten Arzneimitteln in der neonatologischen Intensivmedizin. Klin Padiatr 2009; 221: 275-277

8 Simon A, Ammann RA, Wilkesmann A et al. DSM RSV Paed. Study Group. Respiratory syncytial virus infection in 406 hospitalized premature infants: results from a prospective German multicentre database. Eur J Pediatr 2007; 166: 1273-83

9 Simon A, Nowak H, Sterz R. Use of Palivizumab in Germany: Data from 2002-2007. Klin Padiatr 2011; 223: 1-8

10 Ziegler B, Straßburg H-M. Impfstatus bei Frühgeborenen mit einem Geburtsgewicht unter $1500 \mathrm{~g}$ im Alter von 2 Jahren - eine deutschlandweite Piloterhebung. Klin Padiatr 2010; 222: 243-247 